\title{
Tipo de entrenamiento y momento de elaboración de descripciones poscontacto contingencial en tareas de igualación de la muestra
}

\author{
GERARDO ORTIZ \\ Centro de Estudios e Investigaciones en Comportamiento, Jalisco, México \\ ORCID: https://orcid.org/0000-0002-8290-2176 \\ MARÍA CONCEPCIÓN CISNEROS \\ Universidad de Guadalajara, Jalisco, México \\ ORCID: https://orcid.org/0000-0002-2643-5761 \\ LUIS HERNANDO SILVA \\ Universidad de Guadalajara, Jalisco, México \\ ORCID: https://orcid.org/0000-0001-6363-0083
}

\begin{abstract}
How to quote this article: Ortiz, G., Cisneros, M.C. \& Silva, L.H. (2019). Tipo de entrenamiento y momento de elaboración de descripciones poscontacto contingencial en tareas de igualación de la muestra. Acta Colombiana de Psicología, 22(1), 202-215. doi: http://www.dx.doi.org/10.14718/ ACP.2019.22.1.10
\end{abstract}

Resumen

\begin{abstract}
En el presente trabajo se evaluó el efecto de distintos tipos de entrenamiento sobre (a) la ejecución en pruebas de aprendizaje y transferencia, y (b) la elaboración de descripciones poscontacto en una tarea de discriminación condicional de segundo orden. Con este fin, se asignaron 16 participantes a cuatro grupos; la fase de entrenamiento para los grupos 1 y 3 consistió en la observación de una tarea de discriminación condicional de segundo orden en la que las respuestas de igualación fueron resaltadas con un marco rojo y se indicó, para cada ensayo, si eran acertadas o erróneas (entrenamiento observacional), mientras que para los participantes de los grupos 2 y 4 la tarea consistió en emitir una respuesta explícita de igualación ensayo por ensayo (entrenamiento instrumental). Adicionalmente, se le pidió a los participantes de los grupos 3 y 4 que realizaran una descripción de las contingencias enfrentadas cada doce ensayos, y al finalizar la tarea completa se le pidió a todos los participantes que elaboraran una descripción similar. Como resultado, los participantes de los grupos que estuvieron bajo entrenamiento observacional presentaron el mayor porcentaje de aciertos en las pruebas de aprendizaje y transferencia, además de que elaboraron descripciones poscontacto más específicas y pertinentes que los individuos de los grupos restantes. Estos resultados sugieren que el aprendizaje mediante la observación puede darse incluso si el observador no es expuesto directamente a la ejecución de la respuesta de otro individuo, es decir, aun cuando las respuestas reforzadas se presenten solo de manera gráfica. Palabras clave: entrenamiento observacional, entrenamiento instrumental, descripciones poscontacto, reglas, igualación de la muestra de segundo orden.
\end{abstract}

\section{Training type and moment of elaboration of post-contact descriptions in a matching-to-sample task}

\begin{abstract}
The effects of different types of training over a) performance on learning and transfer tasks, and b) generation of post-contact descriptions in a second order matching-to-sample task were assessed. 16 participants were randomly assigned to one of four experimental groups. During the training phase, the requirement for participants in groups 1 and 3 consisted of the observation of a conditional discrimination task in which matching responses were highlighted with a red frame and indicated, for each trial, whether they were right or wrong (observational training). During this same phase, the task for participants in groups 2 and 4 was to produce, trial by trial, an explicit matching response (instrumental training). Additionally, participants in groups 3 and 4 were required to write a description of the contingencies every twelve trials. After the task, all participants were requested to develop a similar description. Participants of groups that were under observational training had the highest percentage of correct answers on learning and transfer tests. Similarly, they produced more specific rules than individuals from other groups. These results suggest that learning by observation can occur even if the viewer is not directly exposed to responses of another individual, that is, even when reinforced responses are simply presented graphically.

Keywords: observational training, instrumental training, postcontact descriptions, rules, second order matching-to-sample task.
\end{abstract}

\footnotetext{
Francisco de Quevedo 180 Col. Arcos Vallarta Guadalajara, Jalisco 44130, México. Tel.: +52 (33) 377711500 ext. 33315. oruga@cencar.udg.mx
} 


\title{
Tipo de treinamento e momento de elaboraçấo de descriçóes pós-contato contingencial em tarefas de igualaçáo da amostra
}

Resumo

\begin{abstract}
Neste trabalho, foi avaliado o efeito de diferentes tipos de treinamento sobre a execução em testes de aprendizagem e transferência e a elaboração de descrições pós-contato numa tarefa de discriminação condicional de segunda ordem. Com esse objetivo, foram designados 16 participantes a quatro grupos. A fase de treinamento para os participantes dos grupos 1 e 3 consistiu na observação de uma tarefa de discriminação condicional de segunda ordem na qual as respostas de igualação foram ressaltadas com um sinal vermelho e foi indicado, para cada ensaio, se eram acertadas ou errôneas (treinamento observacional). Nessa mesma fase, para os participantes dos grupos 2 e 4, a tarefa consistiu em emitir uma resposta explícita de igualação ensaio por ensaio (treinamento instrumental). Além disso, foi pedido aos participantes dos grupos 3 e 4 que realizassem uma descrição das contingências enfrentadas a cada doze ensaios. Ao finalizar a tarefa, foi solicitado a todos os participantes que elaborassem uma descrição semelhante. Como resultado, os participantes dos grupos que estiveram sob o treinamento observacional apresentaram maior porcentagem de acertos nos testes de aprendizagem e transferência, e elaboraram descrições pós-contato mais específicas e pertinentes do que os indivíduos dos demais grupos. Esses resultados sugerem que a aprendizagem mediante a observação pode acontecer inclusive se o observador não for exposto diretamente à execução da resposta de outro indivíduo, isto é, ainda quando as respostas reforçadas forem apresentadas somente de maneira gráfica.

Palavras-chave: treinamento observacional, treinamento instrumental, descrições pós-contato, regras, igualação da amostra de segunda ordem.
\end{abstract}

\section{Introducción}

Diversos autores han hecho énfasis en la importancia que las descripciones verbales de las contingencias (como el arreglo contingencial entre objetos, personas, consecuencias y relaciones de dependencia que configuran una situación) pueden tener sobre el comportamiento (Baron, Kaufman \& Stauber, 1969; Danforth, Chase, Dolan \& Joyce, 1990; Galizio, 1979). En este sentido, se ha encontrado que cuando los individuos reciben descripciones previas a la exposición a distintas tareas, a manera de instrucciones, tienden a ajustarse de forma más rápida a las contingencias especificadas aun sin haber tenido contacto directo con ellas en ocasiones anteriores (Baumann, Abreu-Rodrigues \& Souza, 2009; LeFrancois, Chase \& Joyce, 1988; GonzálezBecerra \& Ortiz, 2014). Por tanto, el control que ejercen estas descripciones sobre la conducta podría considerarse como un caso de control indirecto, dado que no es necesario que el sujeto experimente las consecuencias de su propio comportamiento para poder ajustarse posteriormente a las relaciones de dependencia referidas en una descripción. Generalmente, se ha denominado a tales antecedentes verbales como reglas o instrucciones, algunas veces, incluso, sin hacer distinción (Andronis, 1991; Baron \& Galizio, 1983; Blakely \& Schlinger, 1987; Cerutti, 1989; Hayes, 1986;
Peláez \& Moreno, 1998; Rosenfarb, Newland, Brannon \& Howey, 1992; Skinner, 1966; Vaughan, 1989).

De acuerdo con diversos autores (Ribes, 2000; Ortiz, González \& Rosas, 2008), las instrucciones y las reglas son funciones distintas que pueden adquirir las descripciones sobre las contingencias. Si la descripción contingencial (de la situación o tarea por resolver o resuelta) se da antes de enfrentar la situación y su efecto es reducir el rango de conductas posibles en la situación descrita o enfrentada, entonces se hablaría de descripciones precontacto que adquirieron función instruccional (es decir, una instrucción). También, es posible que aquellos individuos que han logrado hacer frente a una situación o tarea puedan describir verbalmente las contingencias a las que fueron expuestos, abstrayendo (o identificando) ciertos elementos de la situación enfrentada y, eventualmente, seguir dicha descripción precontacto en un momento o situación distinta, por lo cual la descripción adquiriría una función de regla cuando es utilizada como descripción precontacto en una situación distinta y cumple con su función instruccional (Ortiz et al., 2008).

Bajo esta lógica, se ha llevado a cabo una gran cantidad de investigaciones en las que se encuentra una serie de variables que podrían afectar la formulación de descripciones poscontacto contingencial (reglas), y dentro de los factores que se han reconocido como relevantes aparecen 
las diferentes condiciones de exposición a las contingencias, la frecuencia de retroalimentación, la precisión de las descripciones precontacto utilizadas, y el momento en que se solicita la descripción poscontacto, entre otros (González \& Ortiz, 2014; Ortiz \& Cruz-Alaniz, 2011; Ortiz \& González, 2010a; Ortiz, González, Rosas \& Alcaraz, 2006; Silva, Cisneros \& Ortiz, 2014; Vega \& Peña, 2008).

Un aspecto importante sobre la adquisición de una función de regla por parte de la descripción poscontacto es que esta sea utilizada en un momento posterior por el mismo individuo que la formuló, o por otro(s) individuo(s), como instrucción (es decir, una descripción precontacto con función instruccional) para facilitar el ajuste a situaciones novedosas. En este sentido, si bien una descripción poscontacto incluye elementos de la situación identificados por la persona que la enfrentó como relevantes (abstracción), esta adquirirá su función de regla cuando sea utilizada como descripción precontacto $\mathrm{y}$, de hecho, cuando adquiera una función instruccional para el individuo al que se le presenta (y reduzca el rango posible de respuestas en la situación descrita). Con ello, podría considerarse que tanto instrucciones como reglas (como funciones de descripciones realizadas en distinto momento y, en ocasiones, por distintas personas) implican, al menos en algún momento, en el caso de las reglas, control indirecto por las contingencias.

Por otra parte, el comportamiento bajo el control de descripciones (pre o poscontacto contingencial) no es el único caso de control indirecto por contingencias, ya que es posible que el contacto indirecto con las consecuencias mediante la observación de la ejecución de otros derive en el aprendizaje de las relaciones de dependencia implicadas en una situación y, por tanto, en la adquisición de nuevos repertorios de comportamiento. Sin embargo, por lo general, estos cambios en el comportamiento han sido catalogados como aprendizaje observacional (Catania, 2007) y suelen tener implicaciones importantes para el comportamiento de los individuos, puesto que le permiten a sujetos ingenuos hacer contacto con las contingencias a través de la experiencia de otros, y, así, evitar posibles consecuencias aversivas o facilitar el encuentro con contingencias apetitivas que podrían favorecer la supervivencia. En términos funcionales, este aprendizaje se podría considerar como similar a lo que se ha descrito como control instruccional (Baron \& Galizio, 1983; Galizio 1979), es decir, la adquisición de una función instruccional por parte de descripciones precontacto contingencial (Ortiz et al., 2008).

Ahora bien, en la revisión de literatura en el tema, diversos estudios han encontrado evidencia de aprendizaje por observación, tal es el caso del trabajo de Rehfeldt, Latimore y Stromer (2003), donde se encontró que niños con autismo aprendieron discriminaciones condicionales al observar las ejecuciones correctas de un demostrador; o la investigación de Vega y Peña (2008), donde los participantes fueron expuestos de forma indirecta a las contingencias de una tarea de igualación a la muestra ya fuera mediante la observación de la ejecución de un modelo aprendiz (ejecución realizada por un individuo ingenuo experimentalmente en una tarea de igualación a la muestra) o bien mediante la observación de un modelo experto (ejecución de un individuo que respondía de forma acertada durante toda la tarea), en la cual se encontró que, aun cuando en estos casos no existía un contacto directo con las contingencias, dichos entrenamientos parecieron facilitar los desempeños en la transferencia y en la formulación de reglas verbales, por lo que algunos tipos de entrenamiento observacional podrían fungir como una especie de instrucción que le restringe el rango de respuestas al enseñar al individuo cómo responder correctamente y, por tanto, aumentar las posibilidades de ajustarse a una situación contingencial.

Asimismo, Ribes y Castillo (1998), por su parte, encontraron que después de un entrenamiento observacional en una tarea de igualación de la muestra de segundo orden en la que se proporcionó información escrita acerca de la opción correcta, pero no se pidió ninguna respuesta explícita de igualación, los participantes fueron capaces de identificar mediante repuestas de igualación verbales las relaciones correctas en la fase de posprueba, así como en la de transferencia extramodal e intramodal.

A partir de lo anterior, es posible que tanto el contacto directo con la situación (ejecución instrumental) como la observación de las contingencias (eventos, consecuencias $\mathrm{y}$ relaciones de dependencia que se estructuran para formar una situación) que afectan la ejecución de otros en una tarea pueda tener un efecto sobre el aprendizaje de tales contingencias; e, incluso, es plausible que la sola observación de las relaciones entre los componentes de una contingencia (la relación entre un estímulo discriminativo, la respuesta y su consecuencia) pueda facilitar tanto el ajuste posterior a contingencias similares, como la precisión y pertinencia de descripciones verbales poscontacto contingencial (abstracción de elementos relevantes para el ajuste a la situación). Teniendo esto en cuenta, el presente estudio se diseñó con el fin de investigar el efecto de (a) el tipo de entrenamiento (observacional o instrumental) y (b) la frecuencia de solicitud de elaboración de descripciones poscontacto contingencial durante el entrenamiento sobre el aprendizaje (porcentaje de aciertos en condiciones de entrenamiento), la transferencia (porcentaje de aciertos en pruebas) y el tipo de descripciones poscontacto (especificidad y pertinencia) realizadas por el participante en una tarea de igualación de la muestra de segundo orden. 


\section{Método}

\section{Participantes}

A partir de un muestreo no probabilístico intencional, participaron voluntariamente 16 estudiantes universitarios de 18 a 27 años sin experiencia en tareas de igualación de la muestra, nueve eran estudiantes universitarios que recibieron créditos académicos por su colaboración y siete de ellos habían egresado recientemente del nivel superior. En total, asistieron 30 personas, catorce de las cuales obtuvieron puntajes superiores al $50 \%$ en la sesión de preprueba, por lo cual finalizó su participación en esa instancia del experimento.

\section{Escenario experimental}

Todas las sesiones experimentales se llevaron a cabo en cubículos ubicados dentro de las instalaciones del Centro de Estudios e Investigaciones en Comportamiento de la Universidad de Guadalajara, en México. Cada cubículo tenía una dimensión de $2 \mathrm{~m}$ de largo por $1.5 \mathrm{~m}$ de ancho, contaba con iluminación artificial y natural, y se encontraba equipado con escritorio y silla, así como con una computadora (PC) marca comercial colocada sobre el escritorio.

\section{Materiales e instrumentos}

Para la presentación de la tarea experimental se utilizaron computadoras personales de escritorio marca Lenovo ${ }^{\circledR}$ con procesador i3. La programación de los estímulos, la aplicación de la tarea experimental y la recolección de las respuestas de los sujetos se llevó a cabo mediante el programa Authorware 7.0, con base en la tarea utilizada por Quiroga (2008).

\section{Tarea experimental}

La tarea consistió en una prueba de igualación a la muestra de segundo orden dividida en cuatro fases (preprueba, entrenamiento, aprendizaje y transferencia), que a su vez constó de tres pruebas (extra-instancia, extra-modal y extra-relacional). Cada ensayo de la tarea en las diferentes fases estuvo compuesto por dos estímulos selectores (ES) ubicados en la parte superior de la pantalla, un estímulo muestra (EM) ubicado en la parte central y tres estímulos comparativos (ECo) dispuestos de forma horizontal en la parte inferior.

Las figuras utilizadas tanto en las fases de preprueba, entrenamiento, aprendizaje y transferencia extra-relacional (ER) fueron triángulos, cuadrados y círculos, de color amarillo, azul y rojo. Para la prueba de transferencia extrainstancial (EI) se utilizaron trapecios, rectángulos y rombos, de colores fucsia, verde y rosa; $y$, por último, para la prueba de transferencia extra-modal (EM) las figuras utilizadas fueron siempre círculos de diferentes texturas y tamaños. Los estímulos de segundo orden para todas las fases de la tarea fueron cruces y pentágonos regulares de color café y morado. Los criterios de igualación entrenados y evaluados fueron semejanza e identidad, a excepción de la prueba extrarelacional en donde el criterio señalado por los estímulos de segundo orden fue el de diferencia (véase Tabla 1).

De tal forma, para las respuestas de igualación correctas se consideraron aquellas que se ajustaban a dichos criterios. Por ejemplo, si el criterio de relación vigente en el ensayo (indicado por los ES) era semejanza, la respuesta se consideraría correcta si el ECo elegido compartía una propiedad modal -pero no todas-con el EM (misma forma, pero diferente color; mismo color, pero forma distinta; entre otros, según el caso); y si, por otra parte, la relación criterio era identidad, el ECo considerado como correcto sería aquel

Tabla 1.

Estímulos utilizados (formas y colores) así como relaciones criterio vigentes

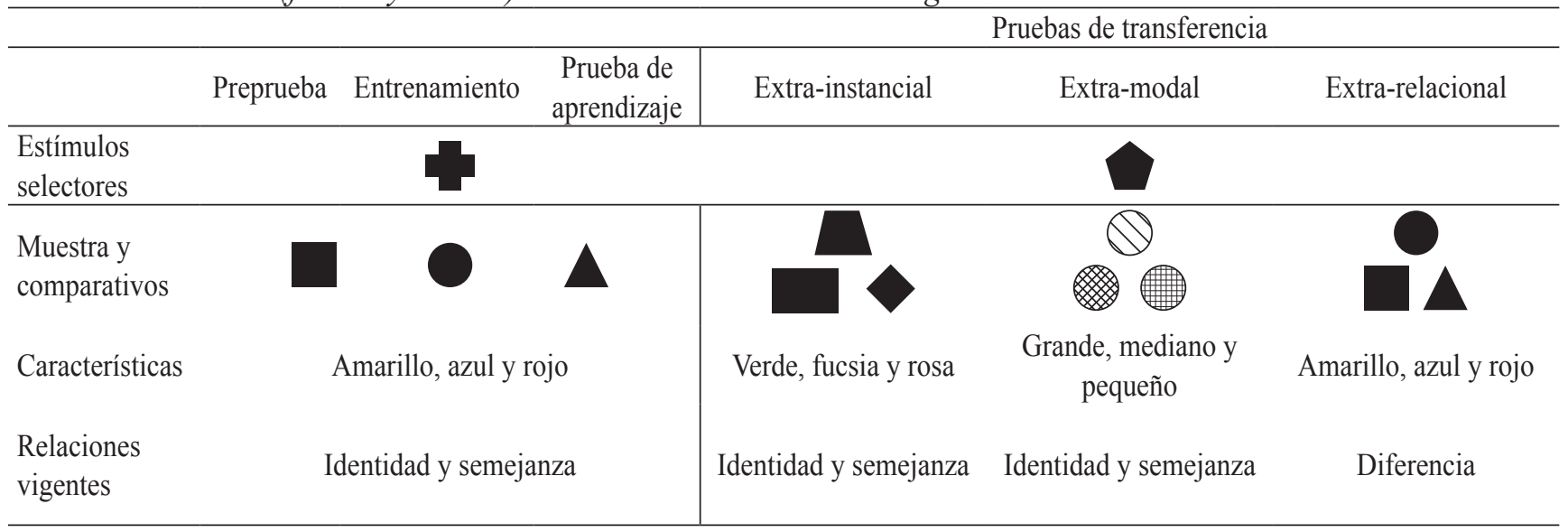


que tuviera propiedades modales iguales al EM (misma forma y color, o mismo relleno y tamaño, según la prueba).

\section{Diseño}

Se utilizó un estudio de comparación intrasujetosentregrupos, con pre y posprueba (prueba de aprendizaje). Los participantes fueron asignados de forma aleatoria a uno de cuatro grupos (véase Tabla 2); y todos enfrentaron una sesión de preprueba, tres de entrenamiento, una de prueba de aprendizaje y tres con diferente tipo de transferencia.

La diferencia entre grupos estribó en el tipo de entrenamiento recibido, resultado de la combinación de los valores de las variables tipo de entrenamiento (observacional vs. instrumental) y frecuencia de descripción poscontacto (una sola vez por sesión vs. dos veces por sesión). Así, mientras los grupos 1 y 3 recibieron entrenamiento observacional, los grupos 2 y 4 enfrentaron entrenamiento instrumental. Al mismo tiempo, se le solicitó a los participantes de los grupos 1 y 2 la descripción poscontacto solo una vez al final de cada sesión (a los 24 ensayos), mientras que a los participantes de los grupos 3 y 4 se les solicitó tal descripción poscontacto dos veces durante cada sesión (cada 12 ensayos).

\section{Procedimiento}

Inicialmente, se invitó a cada sujeto a pasar al cubículo experimental y a sentarse frente al monitor; luego, se les comunicó que se trataba de un estudio sobre procesos generales de aprendizaje, y que no era una prueba de inteligencia; y se dio una descripción precontacto (instrucciones) para el inicio de la prueba (preprueba) igual para todos los grupos:

"En la pantalla aparecerán seis figuras: dos arriba, una en el centro y tres abajo. Debes elegir una de las figuras de abajo, haciendo clic sobre ella. La meta es lograr tantos aciertos como sea posible. Si tienes alguna pregunta, hazla en este momento, pues más adelante no se te podrá suministrar información adicional".

\section{Fase de preprueba.}

Como se dijo anteriormente, todos los sujetos fueron expuestos a la fase de preprueba, de 24 ensayos. Durante esta fase, los criterios de igualación vigentes fueron los de identidad y semejanza, y no existió retroalimentación alguna. El requisito para pasar al bloque de entrenamiento, y continuar con el estudio, fue un desempeño inferior al $50 \%$ de aciertos, debido a que un desempeño superior podría sugerir que los participantes estaban familiarizados con la tarea y podían, por tanto, resolverla de forma adecuada sin entrenamiento.

\section{Fase de entrenamiento.}

Por otra parte, la fase de entrenamiento estuvo dividida en tres bloques de 24 ensayos cada uno y era diferente para cada uno de los grupos. Durante esta fase, para los grupos 1 y 3 el entrenamiento consistió en la observación de una prueba de igualación a la muestra de segundo orden en la que tanto el estímulo muestra como uno de los comparativos se encontraban enmarcados con color rojo (simulando la elección de un participante piloto con un porcentaje de aciertos de 45.8, 66.6 y 91.6 para el primer, segundo y tercer bloque de ensayos, respectivamente) y se indicó en cada ensayo si la elección había sido acertada o errónea. Acá, los participantes estuvieron expuestos tanto a respuestas correctas como a incorrectas, y en estos casos no se requirió una respuesta explícita de igualación (véase Figura 1).

Tabla 2.

Grupos y condiciones experimentales

\begin{tabular}{|c|c|c|c|c|c|c|}
\hline \multirow{2}{*}{$\begin{array}{c}\text { Grupo }(\mathrm{n}=4) \\
1\end{array}$} & \multirow{2}{*}{\multicolumn{2}{|c|}{$\begin{array}{c}\text { Fase de entrenamiento } \\
\text { Observacional } \\
\text { descripción final }\end{array}$}} & \multicolumn{4}{|c|}{ Pruebas de transferencia } \\
\hline & & & & & & \\
\hline 2 & Preprueba & $\begin{array}{c}\text { Instrumental } \\
\text { descripción final } \\
\text { Observacional } \\
\text { descripción c/12 ensayos }\end{array}$ & $\begin{array}{l}\text { Prueba de } \\
\text { aprendizaje }\end{array}$ & $\begin{array}{l}\text { Extra- } \\
\text { instancial }\end{array}$ & Extra-modal & $\begin{array}{l}\text { Extra- } \\
\text { relacional }\end{array}$ \\
\hline 4 & & $\begin{array}{l}\text { Instrumental descripción } \\
\mathrm{c} / 12 \text { ensayos }\end{array}$ & & & & \\
\hline Ensayos & 24 & 72 & 24 & 24 & 24 & 24 \\
\hline Sesiones & 1 & 3 & 1 & 1 & 1 & 1 \\
\hline
\end{tabular}



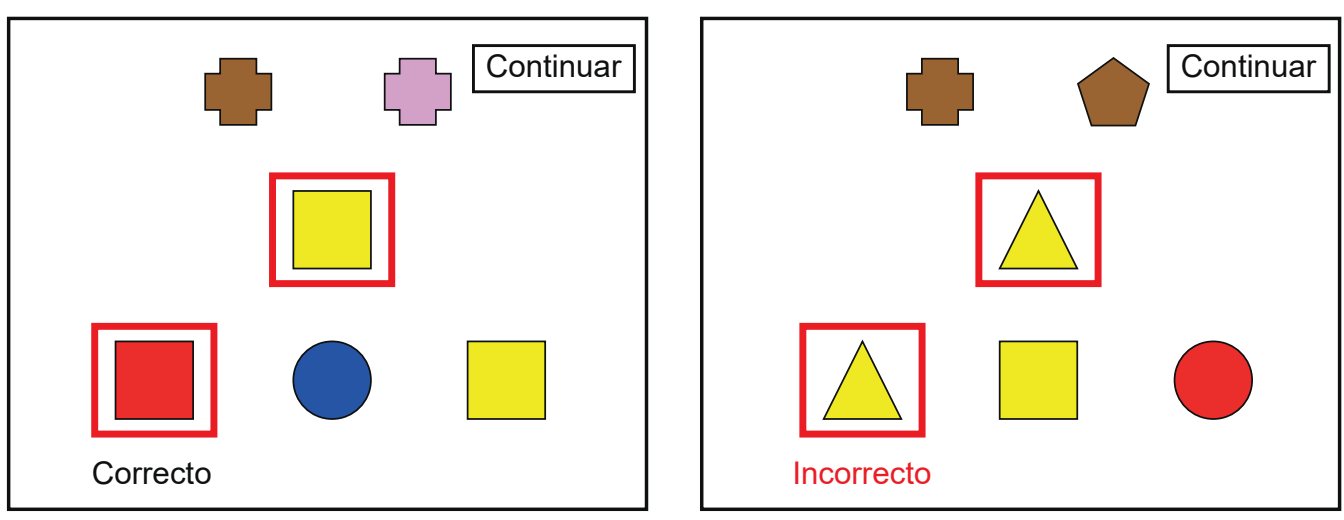

Figura 1. Ejemplos de pantallas para entrenamiento observacional.

Para estos dos grupos, las instrucciones que precedieron (descripciones precontacto) al entrenamiento fueron las siguientes:

"En las siguientes diapositivas te será mostrada una tarea realizada por un individuo. Se han resaltado las respuestas dadas por dicha persona, y se indica, para cada respuesta, si fue acertada o errónea. Intenta observar por qué dichas respuestas fueron calificadas de correctas o incorrectas. Si tienes alguna pregunta, hazla en este momento, pues más adelante no se te podrá suministrar información adicional".

Adicionalmente, se le pidió a cada participante que escribiera los criterios bajo los cuales creía que se había realizado la tarea que había observado. Para los sujetos del Grupo 1 la petición se hizo únicamente al finalizar las pruebas de transferencia, mientras que para los participantes del Grupo 3 la indicación se realizó, además, cada 12 ensayos durante el entrenamiento. En este caso, las instrucciones fueron:

"Utilizando el teclado, describe los criterios o la estrategia que quien resolvió la tarea debió tomar en cuenta para realizarla correctamente".

Por otra parte, para los participantes de los grupos 2 y 4 la fase de entrenamiento consistió en una tarea de igualación a la muestra de segundo orden en la que se pidió la emisión de una respuesta explícita de igualación (de aquí en adelante, entrenamiento instrumental). Durante cada ensayo, cuando la ejecución fue correcta (seleccionar el ECo que correspondía con el Em a partir de la relación de semejanza en forma o en color mostrada por los ES), en la pantalla aparecía un letrero con la palabra "acierto"; en caso contrario, se mostró un letrero con la palabra "error". Las instrucciones para estos grupos fueron:

"En la pantalla aparecerán seis figuras: dos arriba, una en el centro y tres abajo. Dando clic con el mouse debes elegir una de las figuras de abajo que tenga relación con la del centro de acuerdo a lo que indican las de arriba. La meta es lograr tantos aciertos como sea posible. Si tienes alguna pregunta, hazla en este momento, pues más adelante no se te podrá suministrar información adicional".

De igual forma, se le pidió a los participantes de estos grupos que escribieran los criterios bajo los cuales habían realizado la tarea. Para el Grupo 2 esta petición se hizo al terminar las pruebas de transferencia, mientras que para el Grupo 4 la indicación se dio, adicionalmente, cada 12 ensayos. En este caso, las instrucciones fueron:

"Utilizando el teclado, describe los criterios o la estrategia que tomaste en cuenta para realizar la tarea."

\section{Fase de pruebas de aprendizaje y transferencia.}

Las fases de aprendizaje y transferencia fueron las mismas para todos los participantes. Específicamente, la sesión de aprendizaje estuvo conformada por 24 ensayos y los criterios de respuesta fueron identidad y semejanza. Acá, los participantes no recibieron información alguna acerca de si sus respuestas habían sido acertadas o erróneas.

En la fase de transferencia se realizaron tres pruebas diferentes (Varela \& Quintana, 1995): (a) la extra-instancial, en la cual se modificaron las formas y colores de las figuras utilizadas en entrenamiento, mientras que la modalidad (forma y color) y la relación (identidad y semejanza) consideradas correctas se mantuvieron constantes; (b) la extra-modal, en la que se cambiaron las propiedades de los estímulos ante los cuales la elección fue reforzada en entrenamiento (color y forma; durante esta prueba, las propiedades ante las que la elección fue reforzada fueron relleno y tamaño), mientras que las relaciones reforzadas (identidad y semejanza) se mantuvieron constantes; y (c) la extra-relacional, en la que se varió la relación reforzada en entrenamiento (en esta prueba se reforzó el Eco, diferente al EM), pero la forma y color de las figuras utilizadas se mantuvieron 
constantes. Cada una de estas pruebas estuvo compuesta de 24 ensayos (véase Tabla 2). Igual que en la prueba de aprendizaje, no se proporcionó retroalimentación alguna.

\section{Resultados}

La Figura 2 muestra los porcentajes de respuestas correctas por grupo en cada una de las sesiones del estudio. Las barras grises representan el porcentaje promedio de aciertos en prepreuba y prueba de aprendizaje, mientras que los puntos indican los resultados en las sesiones de entrenamiento, y las barras negras señalan los datos en las pruebas de transferencia. Como se puede observar, los participantes de los grupos 2 y 4 tuvieron porcentajes de respuestas acertadas por debajo del $50 \%$ en cada uno de los tres bloques que conformaron el entrenamiento.

En la prueba de aprendizaje se puede observar una ejecución superior al $80 \%$ de aciertos en los grupos 1 y 3 , mientras que los participantes de los grupos 2 y 4 continuaron desempeñándose por debajo del $50 \%$; de hecho, los sujetos del Grupo 4 (instrumental-descripción c/12 ensayos) fueron quienes tuvieron los desempeños más bajos en la prueba de aprendizaje.

En las tres pruebas de transferencia, los participantes del Grupo 3 (observación-descripción cada 12 ensayos) lograron la ejecución con el mayor porcentaje de aciertos, al alcanzar un $97 \%$ de respuestas correctas en la prueba extra-instancia, un $93 \%$ en la extra-modal y un $86 \%$ en la prueba extra-relacional; en estas tres pruebas, los participantes del Grupo 1 (observación) lograron un porcentaje de aciertos de $78 \%$ en la prueba extra-instancia, de $70 \%$ en la prueba extra-modal y un desempeño por encima del $80 \%$ en la prueba extra-relacional; mientras que los participantes del Grupo 2 (instrumental-descripción final) tuvieron ejecuciones que alcanzaron el $50 \%$ de aciertos en la prueba de transferencia extra-instancia, en la prueba extramodal apenas superaron este porcentaje de aciertos, y en la extra-relacional permaneciron por debajo de este porcentaje; y, por último, los sujetos del Grupo 4 respondieron por debajo del $50 \%$ de aciertos en las tres pruebas de transferencia.

Finalmente, en la Figura 3 se puede observar las ejecuciones por sujeto en cuanto al porcentaje de aciertos obtenido
Grupo 1

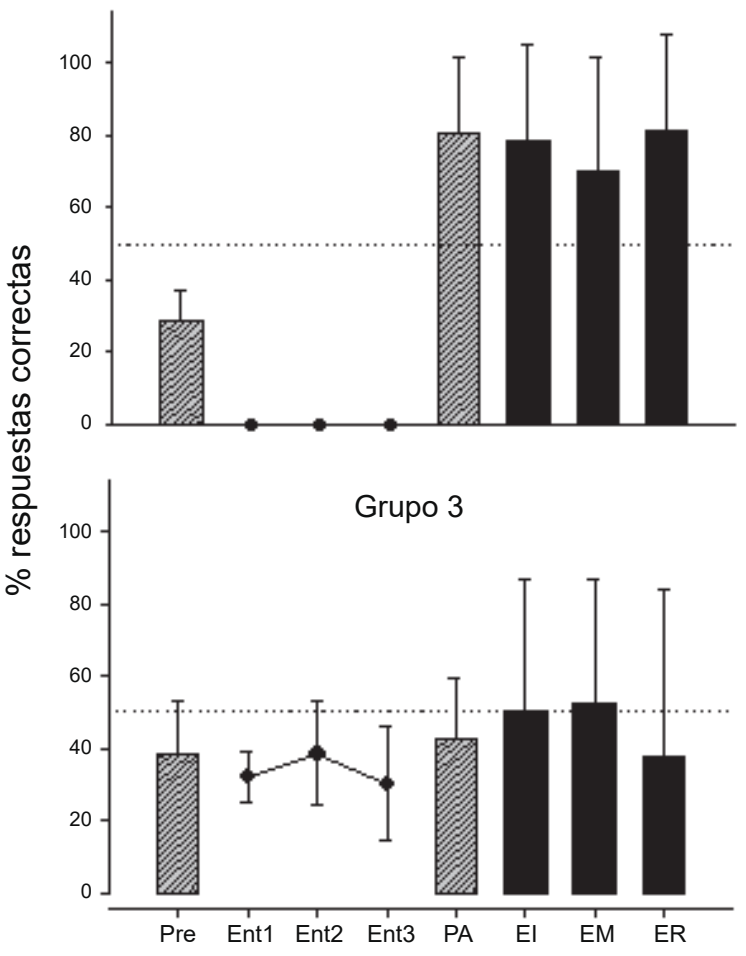

Grupo 2
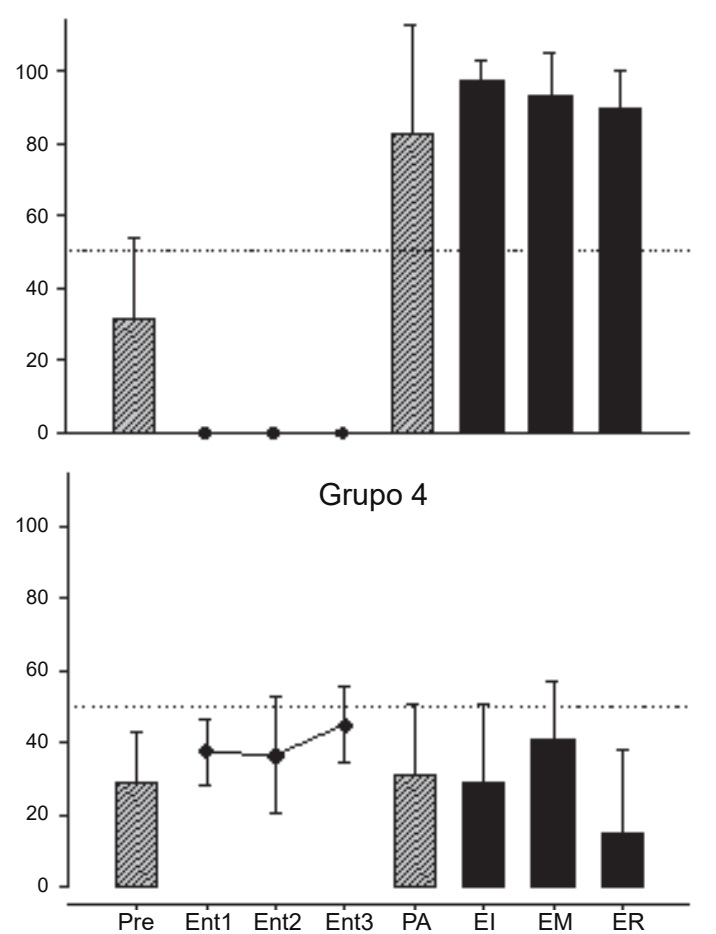

Figura 2. Porcentaje de respuestas correctas, por grupo, en cada una de las pruebas a las que fueron expuestos. 
en cada fase del estudio (el número de aciertos respecto del total de ensayos por sesión), donde resaltan, con respecto a las pruebas de aprendizaje, los participantes 1, 9 y 10, pertenecientes a los Grupos 1 y 3, quienes tuvieron el mejor desempeño al alcanzar el $100 \%$ de respuestas correctas. En contraste, los participantes 14 y 15, pertenecientes al Grupo 2, mostraron las ejecuciones más bajas, al alcanzar solo un porcentaje de aciertos de $4 \%$ y $29 \%$, respectivamente. Y, por último, en las pruebas de transferencia, únicamente los participantes 5 (Grupo 2) y 12 (Grupo 3) alcanzaron el $100 \%$ de respuestas correctas en cada una de ellas, aun cuando en las pruebas de apredizaje solo habían logrado un $62 \%$ y un $33 \%$ de respuestas acertadas, respectivamente.

\section{Análisis de descripciones}

Para analizar las descripciones poscontacto realizadas al finalizar las pruebas de transferencia por los participantes de este estudio se utilizó la taxonomía propuesta por Ortiz et al. (2008). Los autores señalan que una situación de contingencia está conformada por tres componentes: (a) una situación de estímulo, (b) la respuesta del sujeto ante la situación y (c) sus consecuencias; y atribuyen ciertas cualidades a una descripción, como (a) la presencia, (b) la relevancia, (c) la especificidad y (d) la pertinencia. Específicamente, la descripción será calificada como presente si se menciona cualquier o todos los componentes que integran la contingencia -situación de estímulo (SE),
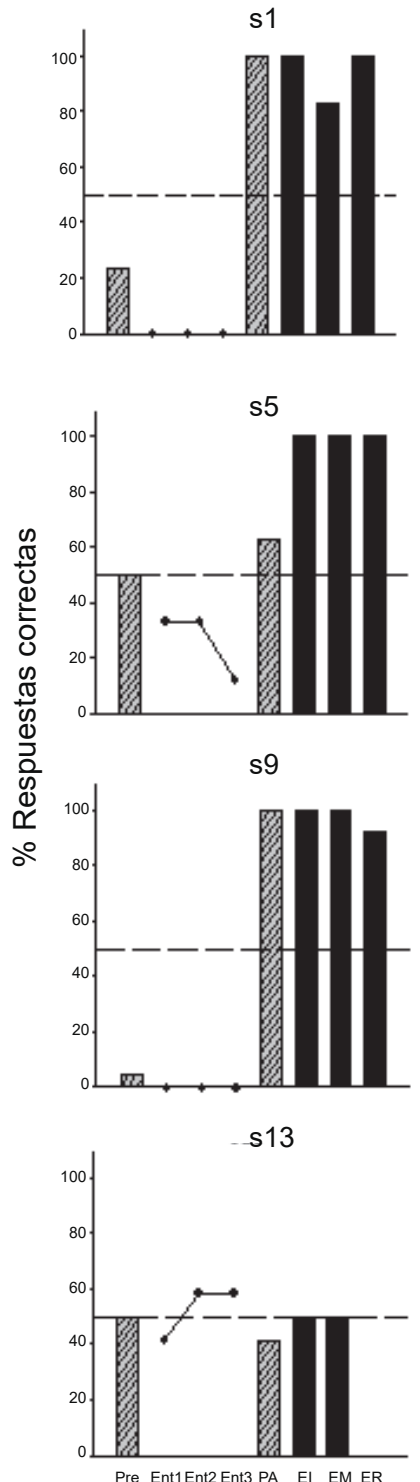

Pre Ent1Ent2 Ent3 PA EI EM ER

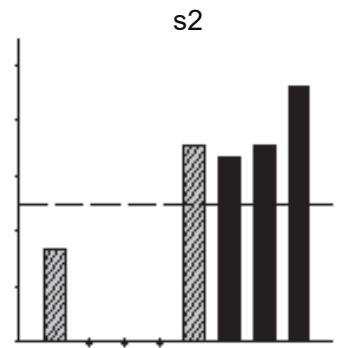

s6
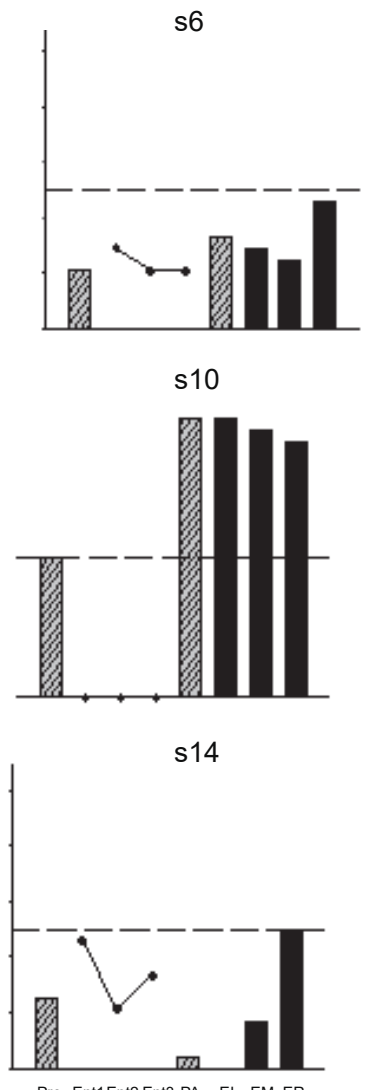

Pre Ent1Ent2 Ent3 PA EI EM ER

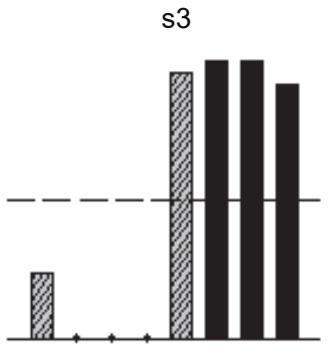

s7
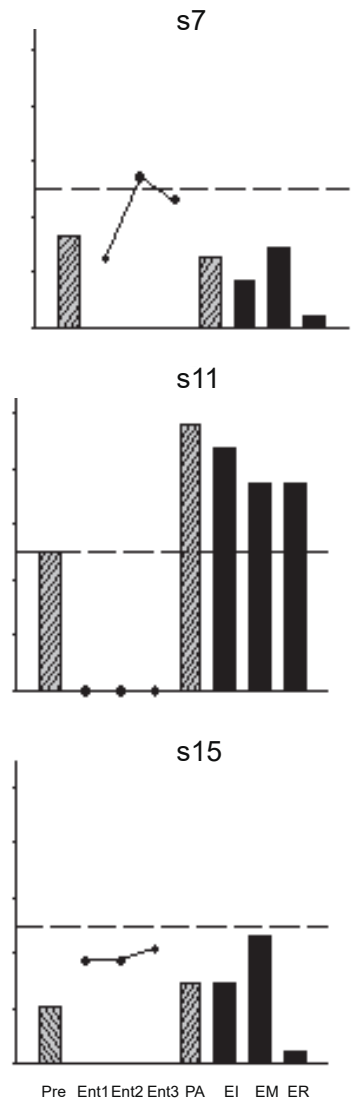

s4

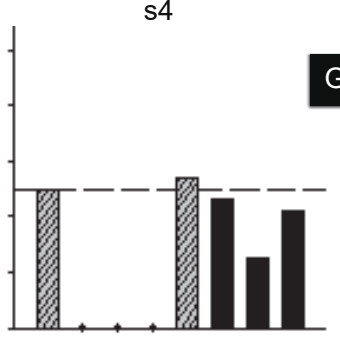

s8
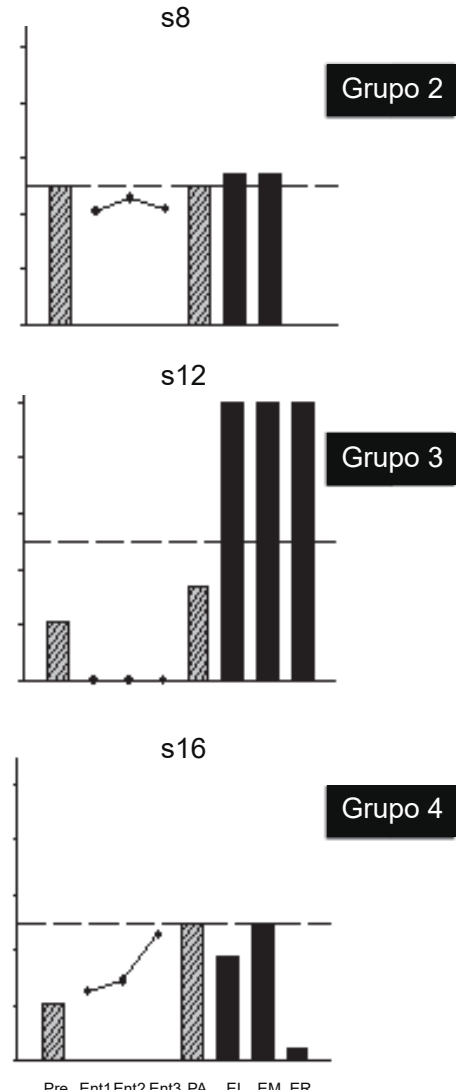

Figura 3. Porcentaje de respuestas correctas por sujeto y por grupo en cada una de las pruebas. 
respuesta (R) y consecuencia (C)-, de otra manera, será calificada como ausente; al adquirir la cualidad de presencia, la descripción puede ser calificada como relevante o irrelevante, dependiendo de si los elementos se encuentran, o no, directamente relacionados con los criterios especificados en un arreglo contingencial; a continuación, si la descripción fue considerada relevante, podrá calificarse como específica o genérica. Si se mencionan todos los elementos relevantes del componente en cuestión (SE, R y C), independientemente de que se incluyan o no elementos irrelevantes, la descripción será calificada como específica; en cambio, si falta la mención de cualquiera de los elementos relevantes, pero se menciona por lo menos uno de ellos, la descripción adquirirá el valor de genérica. De tal forma, entre más específica y pertinente sea una descripción, mejor identificará las relaciones entre los componentes de un arreglo contingencial y los criterios bajo los cuales una respuesta es seleccionada por las consecuencias ambientales en presencia de ciertos estímulos y no de otros.

En consecuencia, en el presente estudio se evaluaron las descripciones relativas a los tres componentes de la situación contingencial: situación de estímulo (SE), respuesta (R) y consecuencia (C), y se analizó si cada uno de ellos se encontraba ausente o presente, y si era relevante o irrelevante, genérico o específico, y pertinente o no pertinente con respecto a la situación que los participantes enfrentaron. Es importante mencionar que si bien las descripciones finales son analizadas con respecto a los tres componentes de la contingencia (SE, R, C), el énfasis se hace, a lo largo del escrito, en cuanto a las descripciones correspondientes al componente Respuesta. Puesto que es en este componente en el que se hace referencia a los criterios utilizados para resolver la tarea, tales descripciones podrían proporcionar más información acerca de las variables que ejercen control sobre el comportamiento.

Por otra parte, en la Figura 4 se muestra el tipo de descripciones poscontacto realizadas al final de la tarea con respecto a cada uno de los componentes descritos anteriormente, indicando el porcentaje de sujetos por grupo que realizaron descripciones ausentes (A), irrelevantes (I), genérico no pertinentes (GNP), específicas no pertinentes (ENP), genéricas pertinentes (GP) o específicas pertinentes (EP).

Con respecto al componente Situación de Estímulo (SE), se puede observar que un alto porcentaje de sujetos, en todos los grupos, realizaron descripciones genéricas pertinentes o específicas pertinentes, lo que significa que la mayoría de los participantes fueron capaces de reconocer y describir varios o todos los elementos de la situación de estímulo a la que se enfrentaron.
Ahora bien, con respecto a los desempeños de los sujetos por grupo al realizar descripciones del componente de Respuesta (R), solo los participantes del Grupo 3 (observacional-descripción c/12 ensayos) realizaron, en un alto porcentaje (75\%), descripciones específicas pertinentes, mientras que la minoría (25\%) realizó descripciones que fueron calificadas como genéricas pertinentes; estas descripciones, calificadas como pertinentes, coinciden con los altos porcentajes de aciertos obtenidos por los participantes de este grupo en las pruebas de aprendizaje y transferencia.

Por otra parte, el $75 \%$ de los participantes del Grupo 4 (instrumental-descripción c/12 ensayos) realizó descripciones genéricas no pertinentes y el $25 \%$ restante, genéricas pertinentes. La elaboración de descripciones poscontacto poco precisas de los participantes de este grupo se corresponde con los bajos porcentajes de aciertos en las pruebas de aprendizaje y transferencia (ninguno de los sujetos que integraron este grupo superó el $50 \%$ de aciertos).

En particular, aun cuando recibieron la petición explícita, tal como los demás participantes, ni el sujeto 1 ni el sujeto 7 (pertenecientes al Grupo 1 y 2 , respectivamente) realizaron descripción alguna; por tanto, los porcentajes en estos dos grupos se obtuvieron únicamente tomando en cuenta a tres participantes; el $33 \%$ de los participantes del Grupo 1 realizó descripciones genéricas no pertinentes, el mismo porcentaje de participantes realizó descripciones genéricas pertinentes y específicas pertinentes; la mayoría de los participantes del Grupo 2 (66\%) realizó descripciones calificadas como genéricas no pertinentes, mientras que el resto de las descripciones fueron calificadas como específicas pertinentes para el componente en cuestión; $y$, finalmente, en las descripciones elaboradas por los participantes de los cuatro grupos, el componente de consecuencia (C) tendió a estar ausente.

Por otra parte, en la Figura 5 se muestra el tipo de descripciones poscontacto realizadas por cada sujeto al finalizar la tarea, evaluadas con respecto a cada uno de los componentes contingenciales de la tarea y de acuerdo con los criterios especificados en la taxonomía propuesta por Ortiz, González y Rosas (2008). De este modo, solo el participante 3, perteneciente al Grupo 1, realizó una descripción poscontacto específica pertinente para cada uno de los componentes de la situación contingencial; y, en correspondencia con el tipo de descripción realizada, puede observarse que este sujeto logró un alto porcentaje de aciertos en pruebas de aprendizaje y transferencia. Por otra parte, el participante 2 de este mismo grupo elaboró una descripción genérica pertinente con respecto al componente de respuesta, lo que coincide con un desempeño superior al $70 \%$ de aciertos obtenido en aprendizaje y un $90 \%$ de aciertos en la última prueba de transferencia. De igual 

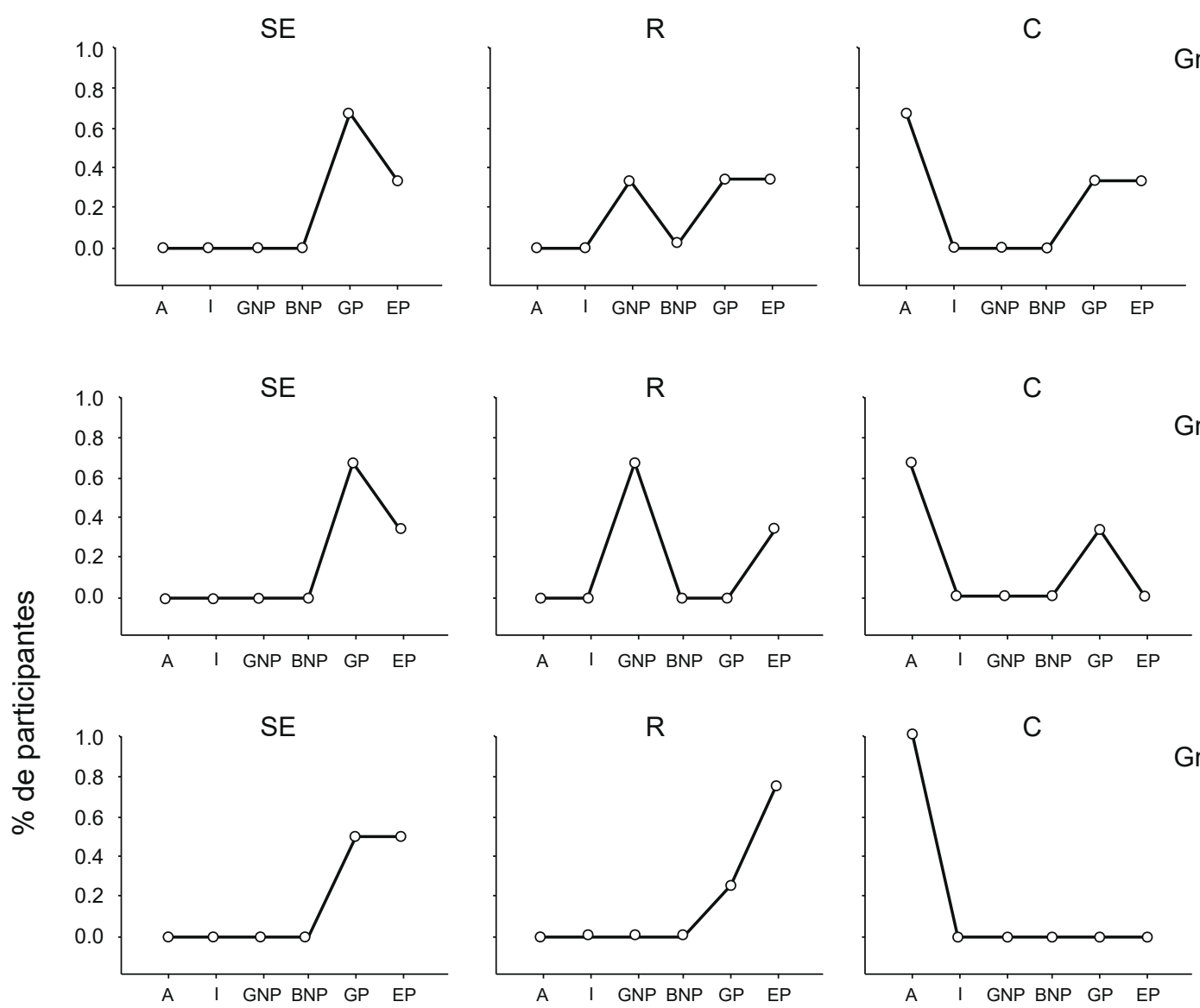

Grupo 1
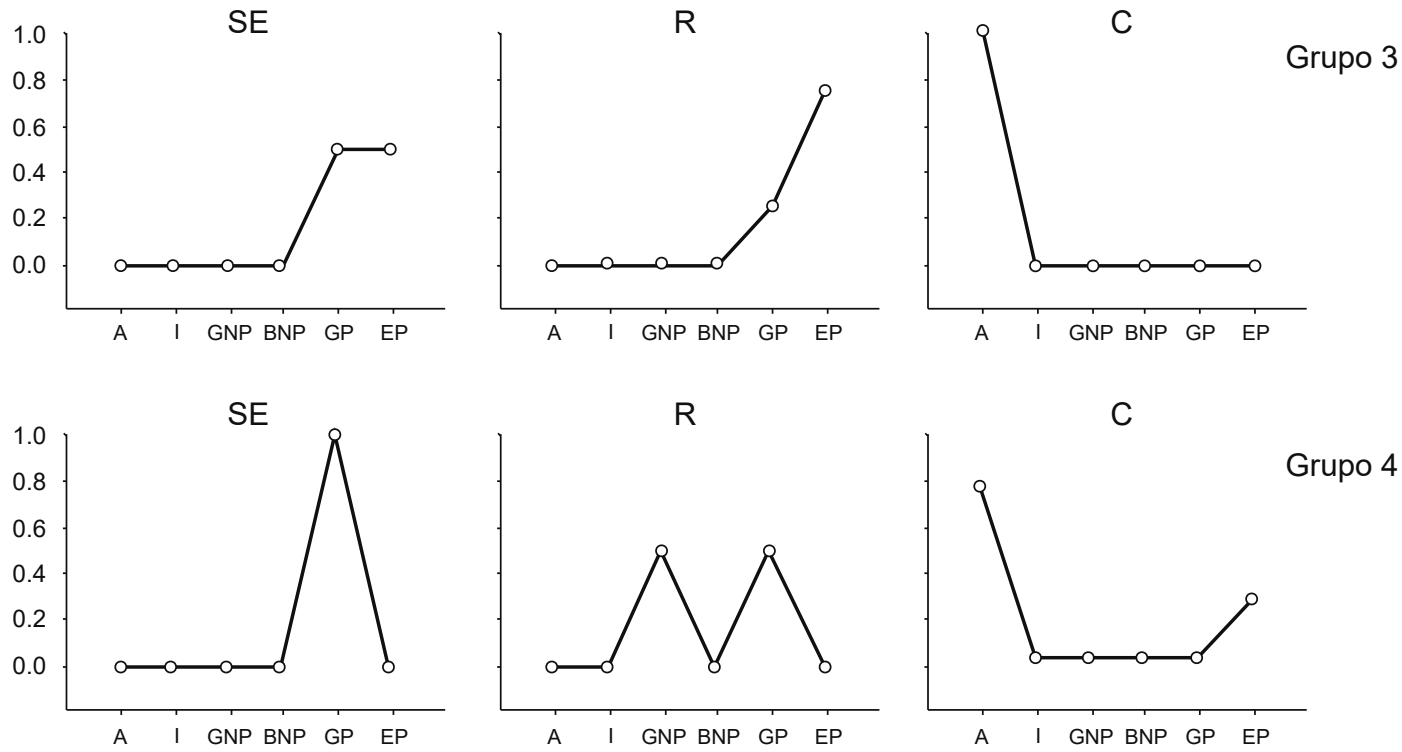

Tipo de descripción

Figura 4. Porcentaje de sujetos cuyas descripciones poscontacto fueron calificadas para cada componente de la situación contingencial (Situación de Estímulo, Respuesta y Consecuencia) como Ausentes (A), Irrelevantes (I), Genéricas no pertinentes (GNP), Específicas o pertinentes (ENP), Genéricas pertinentes (GP) o Específicas pertinentes (EP).

forma, se encuentra relación entre el tipo de descripción realizada por el sujeto 4 (Genérica no pertinente, GNP), que se considera poco ajustada, y el bajo porcentaje de aciertos obtenido en las pruebas posteriores al entrenamiento.

Finalmente, con respecto a los participantes del Grupo 2, se puede observar que el sujeto 5 realizó una descripción del componente de respuesta calificada como específica pertinente, a la vez que obtuvo altos porcentajes de aciertos en las pruebas de transferencia; mientras que los sujetos 6 y 7 realizaron descripciones genéricas pertinentes para el componente de respuesta. También, tres de los participantes del Grupo 3 (participantes 9, 10 y 12) realizaron descripciones específicas pertinentes para el componente de respuesta, mientras que el sujeto restante 

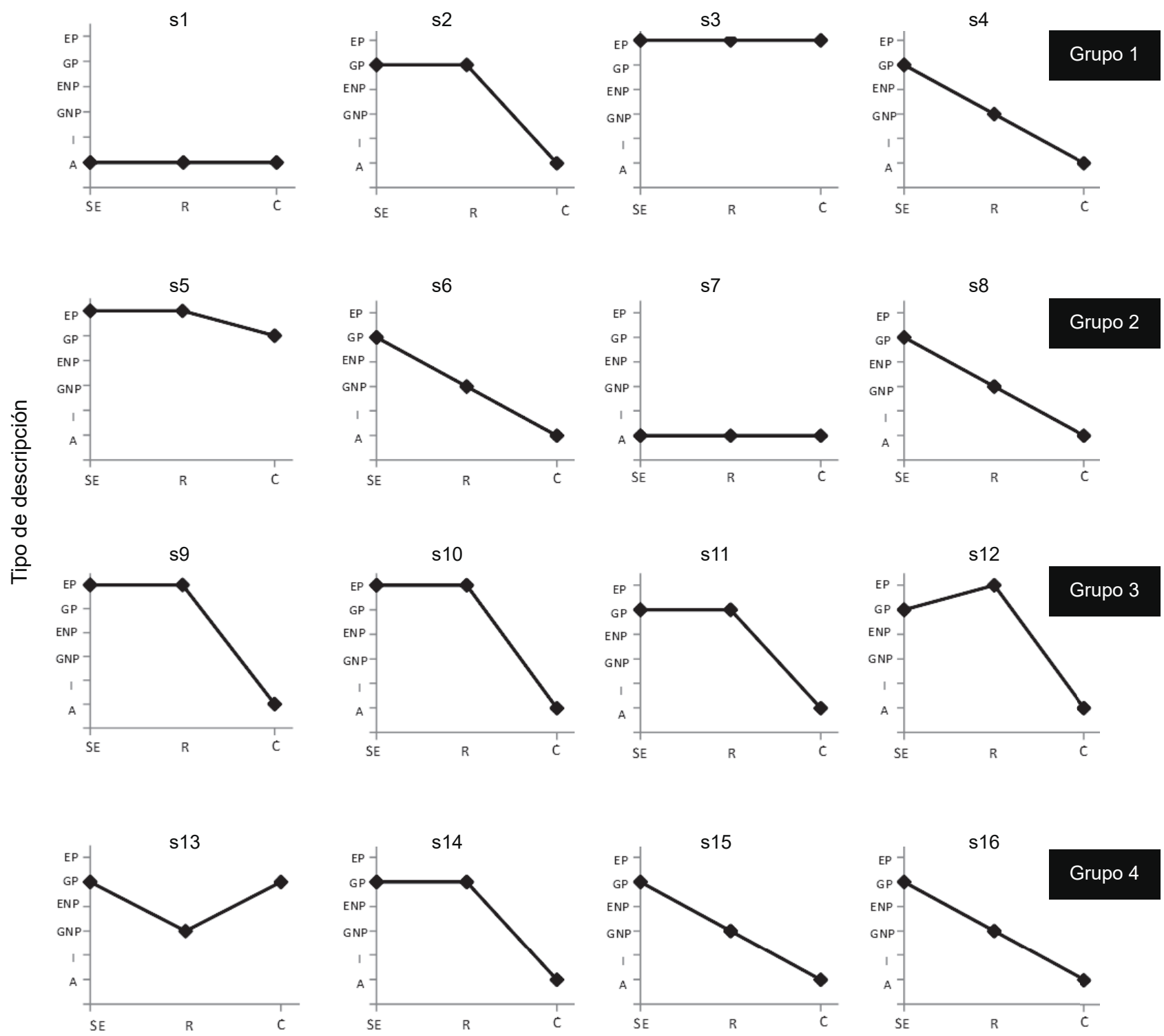

Componentes de la situación de contingencia

Figura 5. Tipo de descripciones poscontacto por sujeto y por grupo, clasificadas para cada componente de la situación contingencial (Situación de Estímulo, Respuesta y Consecuencia) como Ausentes (A), Irrelevantes (I), Genéricas no pertinentes (GNP), Específicas no pertinentes (ENP), Genéricas pertinentes (GP) o Específicas pertinentes (EP).

(sujeto 11) realizó una descripción para este componente que fue calificada como genérica pertinente. Asimismo, las descripciones elaboradas por tres de los participantes del Grupo 4 (sujeto 13, 15 y 16) fueron calificadas como genéricas no pertinentes, $\mathrm{y}$ tales ejecuciones coinciden con un desempeño individual no mayor al $50 \%$ de respuestas correctas en las pruebas de aprendizaje y transferencia. En contraste, el participante número 14, perteneciente a este último grupo, realizó una descripción genérica pertinente para el componente de respuesta aun cuando no superó el $50 \%$ de aciertos en pruebas de aprendizaje y transferencia. 


\section{Discusión}

Con respecto a la relación entre el tipo de entrenamiento y sus efectos en la respuesta de igualación (ejecución del individuo), los datos indican que los sujetos que estuvieron expuestos a entrenamiento observacional (Grupos 1 y 3 ) lograron las ejecuciones con mayor número de aciertos en las pruebas de aprendizaje y transferencia. Estos datos, en concordancia con los hallazgos de algunos autores (Ribes, Barrera \& Cabrera, 1998; Vega \& Peña, 2008), parecen apoyar el supuesto de que exponer a un individuo a un entrenamiento por observación que le permita un contacto con las relaciones que se han estipulado como correctas o incorrectas a partir de un criterio de relación (como semejanza, identidad o diferencia), podría propiciar el control del comportamiento por las contingencias aun sin tener que emitir una respuesta explícita de igualación.

Adicionalmente, los hallazgos de este estudio aportan evidencia de que el aprendizaje mediante la observación puede darse aun si el observador no es expuesto directamente a la ejecución de la respuesta de otro individuo, es decir, que el aprendizaje de las relaciones de contingencia puede presentarse también en arreglos donde las respuestas reforzadas se presentan únicamente de forma gráfica.

Sin embargo, es importante considerar algunos factores particulares al tipo de entrenamiento que pudieran estar ejerciendo un control sobre el comportamiento, como que, en el presente estudio, el tiempo de contacto con los estímulos del arreglo en los cuales se indicaba la elección que se había hecho y la información sobre si esta había sido correcta o incorrecta no tuvo un límite para los participantes que estuvieron expuestos a entrenamiento observacional. En contraste, inmediatamente después de que los participantes a quienes se les solicitó una respuesta explícita realizaban su elección y recibían información acerca de si esta se había considerado acertada o errónea, los estímulos del arreglo y la información sobre la pertinencia de la respuesta desaparecían de la pantalla para comenzar con un nuevo ensayo; de tal forma, para estos individuos el tiempo de contacto con su propia ejecución y las consecuencias de la misma estuvieron reducidos al mínimo, y, por tanto, es posible que el tiempo en el que los sujetos se exponen al arreglo contingencial una vez que la respuesta ha sido emitida y han recibido información sobre si aquélla ha sido acertada o errónea juegue un papel relevante sobre el aprendizaje de nuevas relaciones de dependencia entre los estímulos que conforman una situación.

Por otra parte, algunos autores (Ribes, 2000; Vega \& Peña, 2008) han sugerido que la exposición tanto a instancias correctas como incorrectas en una situación puede ser de importancia para facilitar el control del comportamiento por las contingencias; $\mathrm{y}$, de acuerdo con esto, es posible suponer que el contacto con diferentes porcentajes de respuestas correctas e incorrectas durante el entrenamiento pueda tener algún efecto sobre la ejecución de los participantes en pruebas de aprendizaje y transferencia. En concordancia con lo anterior, en el presente estudio se encontró que mientras que los participantes de los grupos de entrenamiento observacional (quienes tuvieron mejores ejecuciones en pruebas de aprendizaje y transferencia) se expusieron a un $68 \%$ de ensayos de respuestas correctas y a un $32 \%$ de respuestas incorrectas respecto al número total de ensayos de entrenamiento (72), los participantes de los grupos instrumentales (quienes obtuvieron menor porcentaje de aciertos en pruebas de aprendizaje y transferencia), en su mayoría, obtuvieron un porcentaje de respuestas correctas inferior al $40 \%$ a lo largo de las tres fases de entrenamiento. No obstante, la ejecución de algunos participantes hace difícil establecer con mayor precisión el efecto de esta variable, ya que, por ejemplo, aun cuando el participante 4 estuvo expuesto al mismo porcentaje de aciertos y errores que sus pares pertenecientes a los grupos observacionales, su ejecución en pruebas de aprendizaje y transferencia estuvo por debajo del $55 \%$ de aciertos.

Adicionalmente, el participante número 5, quien obtuvo solo un $26 \%$ de respuestas acertadas durante los ensayos de entrenamiento, alcanzó el $100 \%$ de aciertos en pruebas de transferencia. Estas discrepancias dificultan el análisis del papel que puede tener la exposición a instancias acertadas y erróneas en el control de las contingencias sobre el comportamiento. Si bien serán necesarias investigaciones posteriores para explorar el papel de esta variable, una alternativa podría consistir en la inclusión de participantes acoplados, debido a que esto podría asegurar que los sujetos que reciban entrenamiento observacional sean expuestos al mismo porcentaje de respuestas correctas e incorrectas que aquellos a quienes les sea requerida una respuesta explícita de igualación durante esta fase de la tarea. Así también, podría variarse el porcentaje de aciertos y errores a los que diferentes participantes son expuestos durante un entrenamiento observacional semejante al utilizado en este estudio mediante una metodología similar a la utilizada por Silva et al. (2014), que consistió en usar ejecuciones pre-establecidas (en términos del total de aciertos o la elección momento a momento) con el fin identificar si la descripción poscontacto realizada se modificaba a partir del conocimiento del resultado de un participante al que supuestamente tal descripción se presentaba como instrucción.

Un dato interesante es el encontrado en la ejecución de los participantes 5 y 12, quienes tuvieron desempeños por abajo del $50 \%$ de aciertos en las pruebas de aprendizaje y, sin embargo, lograron obtener el total de respuestas correctas 
en las tres pruebas de transferencia. Para explicar tales ejecuciones es posible suponer que, en un primer momento, únicamente el estímulo muestra y los comparativos hayan ejercido control sobre la conducta de estos participantes. Dado que en las tareas de igualación a la muestra de segundo orden el criterio de igualación se encuentra especificado por los estímulos muestra, en cada uno de los ensayos el desempeño en pruebas transferencia pudo haberse favorecido por un contacto tardío con tales estímulos y por la identificación consecuente de los criterios de respuesta. No obstante, las variables determinantes para este tipo de ejecuciones deberán ser establecidas en experimentos subsiguientes.

En cuanto a la elaboración de descripciones poscontacto, los datos sugieren que el tipo de entrenamiento utilizado tuvo un efecto sobre el tipo de descripción formulada, ya que, como se indicó anteriormente, los participantes de los grupos observacionales lograron, en general, elaborar descripciones más pertinentes y específicas, mientras que aquellos de los grupos restantes tendieron a elaborar descripciones más genéricas y menos pertinentes.

No obstante, la descripción elaborada por el sujeto 4 (calificada como genérica no pertinente en el componente de respuesta), quien estuvo expuesto a entrenamiento observacional, y aquella elaborada por el participante 5 (calificada como específica pertinente en el componente de respuesta), a quien le fue requerida una respuesta explícita de igualación durante el entrenamiento, sugieren que más que del tipo de entrenamiento utilizado, la precisión de las descripciones poscontacto contingencial podría depender de la propia ejecución (González-Becerra \& Ortiz, 2014; Guerrero-Radillo \& Ortiz, 2007; Ortiz \& Cruz-Alaniz, 2011; Ortiz \& González, 2010b), en tanto que el participante número 4 tuvo un bajo porcentaje de aciertos en general, y el sujeto número 5 logró el $100 \%$ de respuestas correctas en las pruebas de transferencia. Adicionalmente, con excepción de un participante, existió correspondencia entre el tipo de reglas elaboradas y el porcentaje de aciertos obtenido, y esto coincide con lo descrito por Ribes (2000) sobre que solo ejecuciones efectivas en una condición determinada permitirán la formulación de reglas adecuadas como producto del contacto previo con las propiedades de los estímulos que la conforman.

Por otra parte, con respecto al papel que tuvo la elaboración repetida de descripciones durante el entrenamiento sobre la ejecución, parece que esta variable no ejerció un gran efecto. Si acaso, los datos sugieren que este tipo de requerimiento podría interferir con las ejecuciones de los participantes que emiten una respuesta instrumental (fueron estos participantes los que lograron un menor porcentaje de aciertos) y, en contraste, podría facilitar el control de la respuesta por las contingencias en los sujetos que han sido expuestos a un entrenamiento observacional (fueron los participantes del Grupo 3 quienes lograron el porcentaje de aciertos superior). Sin embargo, la gran similitud entre la ejecución de los participantes de los grupos observacionales y entre la de los pertenecientes a los grupos instrumentales dificulta establecer el papel que esta variable podría tener sobre el comportamiento en tareas de igualación.

Finalmente, es importante mencionar que los resultados encontrados en este experimento contrastan con datos arrojados por experimentos anteriores que utilizaron tareas de igualación de la muestra de segundo orden, puesto que en ellos se ha observado que los sujetos logran ejecuciones efectivas en pruebas de aprendizaje después haber sido entrenados de forma instrumental (Ribes \& Castillo, 1998; Ribes, Moreno \& Martínez, 1995; Ribes, Torres \& Ramírez, 1996; Ribes \& Zaragoza, 2008). Es posible que la diferencia entre los hallazgos se deba al tipo de instrucciones utilizadas, a variables individuales concernientes a las poblaciones utilizadas o a otros factores no controlados. Teniendo esto en cuenta, en investigaciones posteriores el esfuerzo por la identificación de variables que pudieran ejercer algún control sobre la ejecución en este tipo de tareas será de gran importancia para alcanzar mayor generalización en los resultados.

\section{Referencias}

Andronis, P. (1991). Rule governance: Enough to make a term mean. En L. J. Hayes \& P. N. Chase (eds.) Dialogues on verbal behavior (pp. 226-235). Reno, NV: Context Press.

Baron, A., \& Galizio, M. (1983). Instructional control of human operant behavior. The Psychological Record, 33, 495-520.

Baron, A., Kaufman, A., \& Stauber, K. A. (1969). Effects of instructions and reinforcement-feedback on human operant behavior maintained by fixed-interval reinforcement. Journal of the Experimental Analysis of Behavior, 12, 701-712. doi: http://dx.doi.org/10.1901/jeab.1969.12-701

Bauman, A., Abreu-Rodrigues, J., \& Souza da Silva, A. (2009) Rules and Self-Rules: Effects of Variation Upon Behavioral Sensitivity to Change. The Psychological Record, 59, 641670. doi: http://dx.doi.org/10.1007/BF03395685

Blakely, E., \& Schlinger, H. (1987). Rules: Function-altering contingency-specifying stimuli. The Behavior Analyst, 10, 183-187. doi: http://dx.doi.org/10.1007/BF03392428

Cerutti, D. T. (1989). Discrimination theory of rule-governed behavior. Journal of Experimental Analysis of Behavior, 51, 259-276. doi: http://dx.doi.org/10.1901/jeab.1989.51-259

Catania, A. C. (2007). Learning (interim 4th ed.). CornwallonHudson, NY: Sloan Publishing.

Danforth, J. S., Chase, P. N., Dolan, M., \& Joyce, J. H. (1990). The establishment of stimulus control by instructions and by differential reinforcement. Journal of the Experimen- 
tal Analysis of Behavior, 54(2), 97-112. doi: http://dx.doi. org/10.1901/jeab.1990.54-97

Galizio, M. (1979). Contingency-shaped and rule-governed behavior: Instructional control of human loss avoidance. Journal of the Experimental Analysis of Behavior, 31(1), 53-70. doi: http://dx.doi.org/10.1901/jeab.1979.31-53

González-Becerra, V., \& Ortiz, G. (2014). Efectos del tipo y contenido de las descripciones pre-contacto sobre la conducta de discriminación condicional y las descripcionespostcontacto. Acta Colombiana de Psicología, 17(1), 11-23.

Guerrero-Radillo, A. P., \& Ortiz, G. (2007). El papel de la retroalimentación y la ausencia o presencia de instrucciones en la elaboración de descripciones en tareas de discriminación condicional. Acta Colombiana de Psicología, 10(1), 5-13.

Hayes, S. C. (1986). The case of the silent dog-Verbal reports and analysis of rules: A review of Ericsson and Simon's protocol analysis: Verbal reports as data. Journal of the Experimental Analysis of Behavior, 45, 351-363. doi: http:// dx.doi.org/10.1901/jeab.1986.45-351

LeFrancois, J. R., Chase, P. N., \& Joyce, J. H. (1988). The effects of a variety of instructions on human fixed-interval performance. Journal of the Experimental Analysis of Behavior, 49, 383-393. doi: http://dx.doi.org/10.1901/ jeab.1988.49-383

Ortiz, G., \& Cruz-Alaniz,Y. (2011). El papel de la precisión instruccional y la retroalimentación en la ejecución y descripciones poscontacto. Revista Mexicana de Análisis de la Conducta, 37(1), 69-87. doi: http://dx.doi.org/10.5514/ rmac.v37.11.24740

Ortiz, G., \& González, V. (2010a). Efectos de Precisión y Pertinencia del componente Situación de Estímulo de una descripción precontacto. Revista Mexicana de Análisis de la Conducta, 36, 115-130. doi: http://dx.doi.org/10.5514/ rmac.v36.11.18019

Ortiz, G., \& González, V. (2010b). Efecto de dos tipos de descripciones precontacto sobre la ejecución instrumental y descripciones poscontacto en tareas de igualación de la muestra. Acta Colombiana de Psicología, 13(1), 115-126.

Ortiz, G., González A., \& Rosas, M., (2008). Una taxonomía para el análisis de descripciones post contacto con arreglos contingenciales. Acta Colombiana de Psicología, 11(1), 45-53.

Ortiz, G., González, A., Rosas, M., \& Alcaraz, F. (2006). Efectos de la precisión instruccional y de la densidad de retroalimentación sobre el seguimiento instruccional, la elaboración y transmisión de descripciones en tareas de discriminación condicional. Acta Comportamentalia, 14(2), 103-130.

Peláez, M., \& Moreno, R. (1998). A taxonomy of rules and their correspondence to rule-governed behavior. Revista Mexicana de Ananlisis de la Conducta, 24, 197-214. doi: http:// dx.doi.org/10.5514/rmac.v1.i2.27103

Quiroga, L. A. (2008). Aproximaciones metodológicas para el estudio del pensamiento y el lenguaje. Ponencia presentada en el IV Coloquio Internacional de Psicología Interconductual, Lima, Perú.

Rehfeldt, R. A., Latimore, D., \& Stromer, R. (2003). Observational learning and the formation of classes of reading skills by individuals with autism and other developmental disabilities. Reaserch in Developmental Disabilities, 24, 333-358. doi: http://dx.doi.org/10.1016/S0891-4222(03)00059-3

Ribes, E., \& Castillo, A. (1998). Interacción del tipo de entrenamiento y el tipo de respuesta de igualación en transferencia en una discriminación condicional de segundo orden. Acta Comportamentalia, 6, 5-20.

Ribes, E. (2000). Instructions, Rules and Abstraction: a Misconstructed Relation. Behavior and Philosophy, 28, 41-55.

Ribes, E., \& Zaragoza, A. (2008) Efectos de las instrucciones y descripciones con y sin criterio en la adquisición y la transferencia de una discriminación condicional de segundo orden. Acta Comportamentalia, 17, 61-65.

Ribes, E., Barrera, J. A., \& Cabrera, F. (1998). Interacción del entrenamiento observacional en igualación a la muestra de primer orden con el tipo de retroalimentación y respuesta de igualación durante las pruebas de transferencia. Revista Mexicana de Análisis de la Conducta, 24, 339-352.

Ribes, E., Moreno, D., \& Martinez, C. (1995). Efecto de distintos criterios verbales de igualación en la adquisición y transferencia de una discriminación condicional de segundo orden en humanos. Acta Comportamentalia, 3, 27-54.

Ribes, E., Torres, C., \& Ramírez, L. (1996). Efecto de los modos de descripción en la adquisición y transferencia de una discriminación condicional en humanos adultos. Acta Comportamentalia, 4, 159-179.

Rosenfarb, I. S., Newland, M. C., Brannon, S. E., \& Howey, D. S. (1992). Effects of self-generated rules on the development of schedule-controlled behavior. Journal of the Experimental Analysis of Behavior, 58, 107-121. doi: http:// dx.doi.org/10.1901/jeab.1992.58-107

Silva, L., Cisneros, M.C., \& Ortiz, G. (2014). Hice, describí, seguiste, supe tu ejecución: ¿cambia lo que describo? Suma Psicológica, 21, 1, 8-18.

Skinner, B. F. (1966). An operant analysis of problem solving. En B. Kleinmuntz (Ed.). Problem solving: Research, method and theory. (pp. 225-257). New York: John Wiley and Sons.

Varela, J., \& Quintana, C. (1995). Comportamiento inteligente y su transferencia. Revista Mexicana de Análisis de la Conducta, 21, 47-66.

Vaughan, M. (1989). Rule-governed behavior in behavior analysis. En S. C. Hayes (Ed.). Rule-governed behavior: Cognition, contingencies, and instructional control (pp. 97118). EE. UU.: Plenum.

Vega, M., \& Peña, T. (2008) Efecto de diferentes entrenamientos sobre el aprendizaje, transferencia y formulación de la regla en una tarea de igualación a la muestra de primer orden. Revista Colombiana de Psicología, 17, 115-127. 